\title{
Lorentz invariance of basis tensor gauge theory
}

\author{
Edward Bassd* and Daniel J. H. Chung ${ }^{\dagger}$ \\ Department of Physics, University of Wisconsin-Madison, Madison, WI 53706, USA
}

\begin{abstract}
Basis tensor gauge theory (BTGT) is a vierbein analog reformulation of ordinary gauge theories in which the vierbein field describes the Wilson line. After a brief review of the BTGT, we clarify the Lorentz group representation properties associated with the variables used for its quantization. In particular, we show that starting from an $\mathrm{SO}(1,3)$ representation satisfying the Lorentz-invariant U(1,3) matrix constraints, BTGT introduces a Lorentz frame choice to pick the Abelian group manifold generated by the Cartan subalgebra of $\mathrm{u}(1,3)$ for the convenience of quantization even though the theory is frame independent. This freedom to choose a frame can be viewed as an additional symmetry of BTGT that was not emphasized before. We then show how an $S_{4}$ permutation symmetry and a parity symmetry of frame fields natural in BTGT can be used to construct renormalizable gauge theories that introduce frame dependent fields but remain frame independent perturbatively without any explicit reference to the usual gauge field.
\end{abstract}

\section{INTRODUCTION}

Rewriting gauge theories in novel formalisms continue to offer insights into both computational techniques and ideas for physics beyond the SM (see e.g. [1-8]). In analogy with general relativity, ordinary gauge theories of semisimple compact Lie groups (see e.g. [9-15]) can be rewritten in terms of vierbeins of the gauge group space. The quantization of this vierbein theory was called basis tensor gauge theory (BTGT) [16]. Previous works [16-18] focused on a symmetric Lorentz group representation $G_{(f)}^{\mu v}$ of the vierbein field, which transforms as

$$
\left[G_{(f) \beta}^{\alpha}(x)\right]^{i} \rightarrow\left[G_{(f) \beta}^{\alpha}(x)\right]^{j}\left[g^{-1}(x)\right]^{j i}
$$

where $g$ is the representation of the ordinary gauge group. The path integral quantization of $G_{(f)}^{\mu \nu}$ was accomplished using a field redefinition to $\theta_{a}^{B}(x)$ phase variables at the expense of introducing

\footnotetext{
${ }^{*}$ Electronic address: ebasso@wisc.edu

${ }^{\dagger}$ Electronic address: danielchung@wisc.edu
} 
a global field $\left(H^{a}\right)^{\mu v}$ which transforms as a tensor under Lorentz transformations. The Lorentz group representation theory interpretation of $\left(H^{a}\right)^{\mu v}$ is somewhat obscure and the underlying reasons why the global field introduction does not lead to pathologies have not been addressed previously.

In this work, we therefore clarify the Lorentz group representation of $G_{(f)}^{\mu v}$ and its associated representational meaning of this global field $\left(H^{a}\right)_{\mu \nu}$. We show that $G_{(f)}^{\mu \nu}$ is a set of symmetric $S O(1,3)$ complex tensors (closed under the Lorentz transformation orbit before restricting to the $\theta_{a}^{B}(x)$ functional space) satisfying the Lorentz invariant $U(1,3)$ matrix constraints. The set of four $\theta_{a}^{B}$ (for a fixed index $B$ ) parameterizes the $U(1)^{4}$ generated by the Cartan subalgebra of $u(1,3)$. More importantly, we show that $\left(H^{a}\right)_{\mu \nu}$ is a complete set of frame-dependent projections of the usual Lorentz invariant metric $\eta_{\mu \nu}$ which is why there are no global field pathologies. The naive dangers of frame dependence (partly arising from the non-linearity of $G_{(f)}^{\mu \nu}$ map to $\theta_{a}$ ) is argued to be manifestly innocuous because of the reliance of BTGT on the ordinary gauge field $A_{\mu}$ in defining the path integral. Previously stated defining symmetry of the quantized theory (gauge and BTGT invariance) is extended to include frame independence of different choices of $\left(H^{a}\right)_{\mu \nu}$. Finally, we give one recipe for constructing frame independent gauge theories using frame dependent variables based on frame covariance, gauge invariance, BTGT invariance, $S_{4}$ permutation symmetry, and a parity symmetry, without making any explicit reference to the usual gauge field $A_{\mu}$.

The order of presentation is as follows. In Sec. 2, we give a brief review of the BTGT theory. In Sec. 3, we explain how $\left(H^{a}\right)_{\mu \nu}$ is equivalent to $\eta_{\mu \nu}$ and explain how the frame dependent description of frame independent theories arise. An interesting idea in this section is the effect of the non-linearity of the field redefinition (of in going from the $G_{(f)}^{\mu \nu}$ description to $\theta_{a}$ description) on the loss of manifest frame independence. In Sec. 4, we explain how $G_{(f)}^{\mu v}$ forms a set of $S O(1,3)$ tensors satisfying the Lorentz invariant $U(1,3)$ matrix constraints while $\theta_{a}^{B}$ is related to the $U(1)^{4}$ subgroup generated by the Cartan subalgebra of $u(1,3)$. We further explain how the previous symmetries defining the quantized BTGT theory is extended to include frame independence. In Sec. 5 we present a theorem illustrating how one can construct frame independent gauge theories based on frame dependent tensors, using gauge symmetry, BTGT symmetry, $S_{4}$ symmetry, and a parity symmetry, without any explicit reference to the gauge field $A_{\mu}$.

By convention, all repeated indices will be summed unless noted otherwise or is clear from the context of the two sides of the equation. This notational issue should be kept in mind because parts 
of the paper will explain the relationship between the non-manifestly-covariant representations and covariant representations, and this summation convention applies to noncovariant indices as well.

\section{A BRIEF REVIEW OF BTGT}

Let's briefly review the BTGT theory. For an explanation of many statements made in this section, see Refs. [16-18].

Suppose $\phi$ is a matter field which has the following gauge transformation property

$$
\begin{gathered}
\phi^{k}(x) \rightarrow[g(x)]^{k s} \phi^{s}(x) \\
{[g(x)]^{k s} \equiv\left(e^{i \Gamma^{C}(x) T^{C}}\right)^{k s}}
\end{gathered}
$$

where $T^{C}$ are the Hermitian generators satisfying the usual Lie algebraic relationship $\left[T^{A}, T^{B}\right]=$ $i f^{A B C} T^{C}$. The covariant derivative associated with this matter field has a connection, usually expressed as a Lorentz vector field, which tells how to define parallel transports keeping the "gauge direction" parallel as the field is transported along a curve. An alternate formulation of this type of mathematics, typically employed in general relativity to import the spinor technology, involves defining a set of local direction fields having tensor indices in gauge space as well as Minkowski space with which one can use to convert directions in the gauge space into directions in Minkowski

space. With these direction fields, called vierbein fields, one can reproduce the information contained in the original connection field commonly denoted as $A_{\mu}^{C} T^{C}$.

One such choice for the gauge vierbeins is $G_{(f) \beta}^{\alpha}(x)$, which is a complex Lorentz tensor field that transforms as an $\bar{R}$ from the right under the gauge group representation and as a rank 2 Lorentz projection tensor. It has the properties of a gauge vierbein in that

$$
\left[G_{(f) \beta}^{\alpha}(x)\right]^{i} \rightarrow\left[G_{(f) \beta}^{\alpha}(x)\right]^{j}\left[g^{-1}(x)\right]^{j i},
$$

and it essentially maps the gauge space to a Lorentz tensor space, at least locally. The analogy with gravitational vierbeins $\left(e_{a}\right)_{\mu}$ is the following: the indices $\{f, \alpha, \beta\}$ are the analogs of the fictitious Minkowski space index $a$ of $\left(e_{a}\right)_{\mu}$, and the representation of Eq. (4) is the analog of the diffeomorphism acting on the $\mu$ index of $\left(e_{a}\right)_{\mu}$. Explicitly, it allows one to express the gauge field as

$$
A_{\mu}=i\left[G^{-1 \alpha \beta}\right]\left[\partial_{\alpha} G_{\beta \mu}\right]
$$


where $G_{\beta \mu}$ are related to the basis tensor as

$$
\left[G_{\beta \mu}\right]^{q m}=\sum_{f}^{\operatorname{dim} R} \xi_{(f)}^{q}\left[G_{(f) \beta \mu}\right]^{m}
$$

where $\xi_{(f)}^{k}$ are constant vectors that span the gauge group representation $R$ space as

$$
\delta^{k l}=\sum_{f}^{\operatorname{dim} R} \xi_{(f)}^{k} \xi_{(f)}^{* l}
$$

Quite interestingly and suggestively, Eq. (5) is in the form of a sigma model.

Now, we introduce the variables that will be used to pose the question of this paper. To quantize the theory, previous works [16-18] used the following representation involving a symmetric tensor $\left[G_{\beta \mu}\right]^{q m}:$

$$
\left(\left[G_{(f)}(x)\right]_{\delta}^{\gamma}\right)^{j}=\xi_{(f)}^{* l}\left[\left(\exp \left[-i \sum_{a=0}^{3} \theta_{a}^{M}(x) H^{a} T^{M}\right]\right)_{\delta}^{\gamma}\right]^{l j}
$$

or equivalently

$$
G_{\delta}^{\gamma}=\left(\exp \left[-i \sum_{a=0}^{3} \theta_{a}^{M}(x) H^{a} T^{M}\right]\right)_{\delta}^{\gamma}
$$

where $\left(H^{a}\right)^{\mu \nu}$ is a global real field which transforms as a symmetric Lorentz tensor and $\theta_{a}^{M}(x)$ is a real Lorentz scalar field. In practice, one can choose $H^{a}$ explicitly as

$$
\left(H^{a}\right)^{\mu v}=\sum_{b} \psi_{(a)}^{\mu} \psi_{(b)}^{v} \eta^{a b}=\frac{\psi_{(a)}^{\mu} \psi_{(a)}^{v}}{\psi_{(a)} \cdot \psi_{(a)}}
$$

where $\psi_{(a)}^{\mu}$ are four real 4-vectors satisfying $\psi_{(a)}^{\mu} \psi_{(b) \mu}=\eta_{a b}$, where $\eta_{a b}$ coincides with the components of the Lorentzian metric in Cartesian coordinates. This choice of $\psi_{(a)}^{\mu}$ normalization will be generalized later (see Appendix $\mathrm{A}$ ) but can always be made without loss of generality.

The introduction of variables of Eq. (9) was particularly useful for the Abelian theory, where one can set $T^{M}$ to a real number and $\xi_{(f)}=1$ because the map between the gauge field and $\theta_{a}$ simplifies to

$$
A_{\mu}=\sum_{a}\left(H^{a}\right)^{\lambda}{ }_{\mu} \partial_{\lambda} \theta_{a}
$$

which is linear. In the non-Abelian case, the relationship is nonlinear:

$$
A_{\mu}=i \sum_{a} U_{a}\left(H^{a}\right)^{\lambda}{ }_{\mu} \partial_{\lambda} U_{a}^{\dagger}
$$


where

$$
U_{a} \equiv \exp \left[i \theta_{a}^{A} T^{A}\right] .
$$

The gauge transformation on $\theta_{a}^{A}$ can be written as

$$
U_{a} \rightarrow e^{i \Gamma} U_{a}
$$

where $\Gamma \equiv \Gamma^{B} T^{B}$. Explicitly, Baker-Campbell-Hausdorff formula gives

$$
\theta_{a}^{\prime}=\Gamma+\theta_{a}+\frac{i}{2}\left[\Gamma, \theta_{a}\right]-\frac{1}{12}\left(\left[\Gamma,\left[\Gamma, \theta_{a}\right]\right]+\left[\theta_{a},\left[\theta_{a}, \Gamma\right]\right]\right)+\ldots
$$

where $\theta_{a} \equiv \theta_{a}^{A} T^{A}$.

One of the most important new features of a theory with $\theta_{a}$ is that to reproduce ordinary gauge theories, there needs to be a new continuous symmetry which can be referred to as BTGT symmetry $^{1}$ :

$$
U_{a} \rightarrow U_{a} e^{i Z_{a}}
$$

where $Z_{a} \equiv T^{B} Z_{a}^{B}$ satisfies

$$
\left(H^{a}\right)^{\lambda}{ }_{\mu} \partial_{\lambda} Z_{a}=0
$$

and $U_{a}$ is defined in Eq. (13). Note that even though this is a continuous symmetry similar to a gauge symmetry, it is a symmetry without any compensating fields transforming as in gauge transforms. Its main purpose is to package $\theta_{a}^{B}$ into $A_{\mu}$ without any other observables that depend on $\theta_{a}^{B}$ alone. Without this symmetry (or something similar), there would be the usual problems associated with higher derivative theories stemming from Eq. (11) (with a finite power truncation in higher derivatives) and/or global gauge charge violations. It is interesting to note that this BTGT symmetry can be expressed in terms of a transformation of $G^{\mu}{ }_{v}$ independently of the decomposition in $H^{a}$. However, we will defer the exploration of this topic to a future work.

If one solves Eq. (12) for $\theta_{a}^{A}$, it will depend on the boundary conditions. Eq. (16) can be viewed as defining an equivalence relation $\theta_{a}^{A(1)} \sim \theta_{a}^{A(2)}$ where the (1) and (2) superscripts specify different boundary conditions to Eq. (11). Indeed, instead of defining specific transformations such as Eq. (16), one can define a generalized BTGT invariance to be the set of transformations that leave Eq. (12) invariant. Given a curve parameterized by

$$
x_{(c)}^{\mu}(s) \equiv s \psi_{(c)}^{\mu}+x_{0}^{\mu},
$$

\footnotetext{
${ }^{1}$ It is not known whether other symmetries that have the same desired effect exists. Indeed, one way to interpret this paper is to clarify and add to this symmetry, as we explain later.
} 
one can integrate Eq. (12) to obtain

$$
U_{c}^{\dagger}\left(x_{(c)}(s)\right)=U_{c}^{\dagger}\left(x_{(c)}\left(s_{i}\right)\right) \bar{P}\left[\exp \left(-i \int_{s_{i}}^{s} d \tau A_{\mu}\left(x_{(c)}(\tau)\right) \psi_{(c)}^{\mu}\right)\right],
$$

where $\bar{P}$ denotes anti-path-ordering. Exponential of $\theta_{a}$ is clearly is related to the Wilson line.

The action for the BTGT theory is of the form

$$
\mathscr{L}=\frac{-1}{4 g^{2} T(R)} \operatorname{Tr}\left(F^{\mu v} F_{\mu v}\right),
$$

where

$$
-i F_{\mu \nu}=\partial_{\mu}\left[\left(G^{-1}\right)^{\lambda}{ }_{\kappa} \partial_{\lambda} G^{\kappa}{ }_{\nu}\right]-\partial_{\nu}\left[\left(G^{-1}\right)^{\lambda}{ }_{\kappa} \partial_{\lambda} G^{\kappa}{ }_{\mu}\right]+\left[\left(G^{-1}\right)^{\lambda}{ }_{\kappa} \partial_{\lambda} G^{\kappa}{ }_{\mu},\left(G^{-1}\right)^{\rho}{ }_{\alpha} \partial_{\rho} G^{\alpha}{ }_{\nu}\right] .
$$

In terms of $\theta_{a}$ variables (embedded into $U_{a}$ ), this is

$$
\begin{aligned}
-i F_{\mu \nu}= & \partial_{\mu}\left(\sum_{a=0}^{3} U_{a}\left(H^{a}\right)^{\lambda}{ }_{\nu} \partial_{\lambda} U_{a}^{\dagger}\right)-\partial_{\nu}\left(\sum_{a=0}^{3} U_{a}\left(H^{a}\right)^{\lambda}{ }_{\mu} \partial_{\lambda} U_{a}^{\dagger}\right) \\
& +\left[\sum_{a=0}^{3} U_{a}\left(H^{a}\right)^{\lambda}{ }_{\mu} \partial_{\lambda} U_{a}^{\dagger}, \sum_{b=0}^{3} U_{b}\left(H^{b}\right)^{\rho}{ }_{\nu} \partial_{\rho} U_{b}^{\dagger}\right]
\end{aligned}
$$

where we have used

$$
G^{\lambda}{ }_{v}=\sum_{a=0}^{3}\left(H^{a}\right)^{\lambda}{ }_{v} U_{a}^{\dagger}
$$

The introduction of the $\theta_{a}$ variables (through $U_{a}$ and $H^{a}$ ) is useful for path integral quantization.

In the next section, we explain one of the main points of this paper which is to explain how the theory does not have a preferred frame despite the appearance of $\left(H^{a}\right)^{\lambda}{ }_{\mu}$.

\section{WHAT IS $\left(H^{a}\right)^{\lambda \mu}$ ?}

One obvious question arises as to the interpretation of $\left(H^{a}\right)^{\lambda \mu}$ transforming as a symmetric Lorentz tensor. For example, one might very naively guess that $\theta_{a}^{A}\left(H^{a}\right)^{\lambda \mu}$ which enters as a package indicates that it is similar to how $\bar{\Psi} \gamma^{\mu} \Psi$ in spinor representation (say for a Dirac field $\Psi)$ transforms as a Lorentz vector. Recall that in the case of $\bar{\Psi} \gamma^{\mu} \Psi$, the field $\Psi$ transforms as a spinor inducing the behavior as if $\gamma^{\mu}$ transforms. This means, we can always keep $\gamma^{\mu}$ to be the same set of numbers in any Lorentz frame. Indeed, $\Psi$ field has 4 real functional degrees of freedom to encode the Lorentz 4-vector representation.However, a symmetric Lorentz tensor field that is not traceless has 10 functional degrees of freedom, whereas $\theta_{a}^{B}\left(H^{a}\right)^{\lambda \mu}$ for a fixed $B$ has 
only 4 functional degrees of freedom. Hence, the object $\left(H^{a}\right)^{\lambda \mu}$ must transform under Lorentz transformations independently of $\theta_{a}^{B}$. This means that the situation is not analogous to the spinor representation and $\theta_{a}^{B}$ degrees of freedom are frame dependent fields. We clarify the precise covariant representational meaning of $\left(H^{a}\right)^{\lambda \mu}$ in this section.

To see what $\left(H^{a}\right)^{\mu v}$ means, first note that it can be written in terms of $\psi_{(a)}^{\mu}$ according to Eq. (10) which means that its 10 global degrees of freedom are in the constrained set of real 4-vectors $\psi_{(a)}^{\mu}$. We will now show that $\psi_{(a)}^{\mu}$ is just a set of frame-choice induced projections of ordinary tensors used in constructing Lorentz invariant field theories.

In ordinary field theory description, a global field such as $\psi_{(a)}^{\mu}$ naively seems exotic. However, for every choice of coordinates $x^{\mu}[n]$ (where "[n]" labels a particular coordinate system), one implicitly defines in ordinary field theory the following global field

$$
\Delta=\Delta_{\lambda}^{\mu} e_{\mu}[n] \otimes e^{\lambda}[n] \equiv \delta_{\lambda}^{\mu} e_{\mu}[n] \otimes e^{\lambda}[n]
$$

whose components are Lorentz invariant (although dependent on $e^{\mu}[n]$ in general). In the BTGT theory with symmetric gauge vierbeins $G^{\mu v}$ defined in Eq. (6), we first introduce a coordinate frame "[1]" which can be chosen arbitrarily since the vacuum $\left(\theta_{a}=\Gamma\right)$ is Lorentz invariant. We then define $\psi_{(a)}^{\mu}$ as the following projection of the $\Delta$ tensor:

$$
\psi_{(a)}^{\mu} e_{\mu}[n] \equiv \Delta_{\lambda}^{\mu} e_{\mu}[n] \otimes e^{\lambda}[n]\left(e_{a}[1]\right)
$$

where $e_{a}[1]$ refers to an $a$ th basis object of an arbitrarily chosen frame "[1]" whose coordinate basis is spanned by $e_{\mu}[1]$ and the "(...)" denotes the usual dual space defined projection

$$
e^{\lambda}[1]\left(e_{\mu}[1]\right)=\delta_{\mu}^{\lambda} .
$$

This means that as long as we choose $\underline{4}$ independent objects $e_{a}[1], \psi_{(a)}^{\mu}$ can be used to construct $\Delta_{\lambda}^{\mu}{ }_{\lambda}$ which is obviously Lorentz invariant. As we noted, $\Delta$ is used in ordinary field theory. Only difference between an ordinary field theory and the one with $\psi_{(a)}^{\mu}$ is the arbitrary initial frame choice "[1]" and the fact that one can (but do not have to) now describe field effects that prefer a frame "[1]".

For an explicit coordinate dependent expression for $\psi_{(a)}^{\mu}$, consider any frame "[2]" related to frame "[1]" by a Lorentz transform

$$
e^{\lambda}[2]=\Lambda_{\kappa}^{\lambda} e^{\kappa}[1]
$$


We can evaluate Eq. (25) as

$$
\psi_{(a)}^{\mu} e_{\mu}[2]=\Lambda_{a}^{\mu} e_{\mu}[2]
$$

which is the usual basis we used in the BTGT papers. What condition should we place on $\psi_{(a)}^{\mu}$ usage in the path integral to ensure that the theory does not depend on that initial arbitrary frame $x^{\mu}[1]$ used to define $\psi_{(a)}^{\mu}$ ? It is clear that the answer is that the path integral should be rewritable without $\psi_{(a)}^{\mu}$ (and its "a"-index associated tensors) and maintain manifest covariance in having a manifest Lorentz scalar action and have a Lorentz invariant path integral measure. If that were not true, then the physical system would have an observable that probes the properties endowed by the $\psi_{(a)}^{\mu}$ which would mean that an experiment can be done to pick out the arbitrary "[1]" frame with which $\psi_{(a)}^{\mu}$ was defined. We will call this frame independence.

If the local field is a Lorentz tensor whose definition is independent of $\psi_{(a)}^{\mu}$, then $\psi_{(a)}^{\mu}$ should appear in combinations such that it sums to invariant tensors such as $\eta^{\mu \nu}$. For example, if $\phi$ is an ordinary real scalar field whose Lorentz tensor definition is independent of $\psi_{(a)}^{\mu}$, frame independent theories can have $\psi_{(a)}^{\mu}$ only in the combinations such as

$$
\sum_{a b} \eta^{a b} \psi_{(a)}^{\mu} \partial_{\mu} \phi \psi_{(b)}^{v} \partial_{\nu} \phi=\eta^{\mu v} \partial_{\mu} \phi \partial_{\nu} \phi
$$

where the $\psi_{(a)}^{\mu}$ disappears.

However, suppose we define $\left(\psi^{-1} x\right)^{\mu} \equiv\left(\Lambda^{-1}\right)_{\lambda}^{\beta} x^{\lambda}$ where $\psi_{(a)}^{\mu} \equiv \Lambda_{a}^{\mu}$ in accordance with Eq. (28). Let's redefine a vector field

$$
V^{\mu}(x)=\sum_{a} \phi^{(a)}\left(\psi^{-1} x\right) \psi_{(a)}^{\mu}
$$

in terms of Lorentz scalar fields $\phi^{(a)}$ where we clearly see that $\phi^{(a)}$ definition does depend on the definition of $\psi_{(a)}^{\mu}$. Now, as long as $\phi^{(a)}(x)$ is used in the action, we cannot absorb $\psi_{(a)}^{\mu}$ into a coordinate redefinition such that $\psi_{(a)}^{\mu}$ completely disappears and at the same time maintain Lorentz covariance. For example, the Lorentz and frame independent $\int d^{4} x \chi(x) \partial_{\mu} V^{\mu}(x)$ where $\chi(x)$ is a Lorentz scalar becomes

$$
\begin{aligned}
\int d^{4} x \chi(x) \partial_{\mu} V^{\mu}(x) & =\int d^{4} x \sum_{a} \chi(x) \psi_{(a)}^{\mu} \partial_{\mu} \phi^{(a)}\left(\psi^{-1} x\right) \\
& =\sum_{a} \int d^{4} y \chi\left(\sum_{b} \psi_{(b)}^{\lambda} y^{b}\right) \frac{\partial}{\partial y^{a}} \phi^{(a)}\left(y^{c}\right)
\end{aligned}
$$

where we defined

$$
d x^{\mu}=\sum_{b} \psi_{(b)}^{\mu} d y^{b}
$$


and used $d^{4} x=d^{4} y$. Even if we were to remove $\psi_{(b)}^{\lambda}$ in $\chi\left(\sum_{b} \psi_{(b)}^{\lambda} y^{b}\right)$ through a field redefinition of

$$
X(y) \equiv \chi\left(\sum_{b} \psi_{(b)}^{\lambda} y^{b}\right)
$$

what remains is not a manifestly Lorentz invariant expression since $\phi^{(a)}(y)$ is defined to be a scalar under Lorentz transformations. On the other hand, it is clear from Eqs. (30) and (32) that manifest covariance can be recovered if one first considers $\psi_{(a)}^{\mu}=\delta^{\mu}{ }_{a}$ and now treats $a$ index as a covariantly transforming index (i.e. $a$ transforming under the Lorentz group as $\bar{V}^{a}=\Lambda^{a}{ }_{c} V^{c}$ ).

Hence, we see that in situations in which there exists a frame in which frame-dependent indices such as $(a)$ are contracted in a manifestly covariant manner, the theory has no preferred frame as one can rewrite it in a manifestly covariant notation. We will call this frame-covariance. The reason why this occurs is because Eq. (25) tells us

$$
\psi_{(a)}^{\beta}=\Delta_{\lambda}^{\mu} e_{\mu}[1]\left(e_{\beta}[1]\right) \otimes e^{\lambda}[1]\left(e_{a}[1]\right)
$$

which are just components of a $(1,1)$ Lorentz tensor in this defining frame, just as in usual covariant notation where $a$ now behaves as an ordinary Lorentz tensor index.

Next, there is another source of non-covariance besides the frame choice. Although the functional degrees of freedom count between $\theta_{a}$ and $U_{a}$ is the same at least locally, the variable $\theta_{a}$ is different from $U_{a}$ from a frame-covariance representational perspective because it has a non-linear map to ordinary tensor indices unlike the index " $a$ " on $U_{a}$. This non-linearity was introduced to satisfy the gauge group representational requirement of the $G^{\mu v}$ while allowing an unconstrained path integration over $\theta_{a}$. The loss of manifest covariance stems from introducing only the exact number of functional degrees of freedom to match $A_{\mu}$. A more precise representational theory explanation of these "lost" functional degrees of freedom will be given in Sec. 4.

To better understand the nonlinearity that leads to the loss of frame-covariance in terms of $\theta_{a}$, consider the analogous situation of

$$
V^{\mu}(x)=\sum_{a}\left[\Phi^{(a)}\left(\psi^{-1} x\right)\right]^{3} \psi_{(a)}^{\mu}
$$

where $\Phi^{(a)}(x)$ are scalar fields. Eq. (32) turns into

$$
\int d^{4} x \chi(x) \partial_{\mu} V^{\mu}(x)=\sum_{a} \int d^{4} y X(y) \frac{\partial}{\partial y^{a}}\left[\Phi^{(a)}(y)\right]^{3}
$$

where $X$ is defined in Eq. (34). Unlike in Eq. (32), even if $a$ transforms as a Lorentz vector index starting from the $\Lambda=1$ frame, one cannot recover manifest covariance because of the nonlinearity 
of the index appearing in Eq. (36). Furthermore, the loss of frame-covariance also shows up in the path integral measure:

$$
\begin{aligned}
D V & =D \Phi \operatorname{det} \frac{\delta V^{\mu}(x)}{\delta \Phi^{(c)}(y)} \\
& =D \Phi \operatorname{det}_{x y} \operatorname{det}_{\mu a}\left[\Phi^{(a)}\left(\psi^{-1} x\right)\right]^{2} \psi_{(a)}^{\mu} \delta^{(4)}(x-y) \\
& =\mathscr{N}_{1} D \Phi \exp \left[i \int d^{4} y M^{4} \ln \left(\operatorname{det}_{\mu a} 3\left[\Phi^{(a)}(y)\right]^{2} \psi_{(a)}^{\mu}\right)\right]
\end{aligned}
$$

where $\mathscr{N}_{1}$ is a field independent normalization, $M^{4}$ is a momentum space regulator, and we used the change of variables $y=\psi^{-1} x$. Hence, we see that

$$
\begin{aligned}
\int D \chi D V e^{i \int d^{4} x \chi(x) \partial_{\mu} V^{\mu}(x)}= & \mathscr{N} \int D X D \Phi \exp \left[i \int d^{4} y M^{4} \ln \left(\operatorname{det}_{\mu a} 3\left[\Phi^{(a)}(y)\right]^{2} \psi_{(a)}^{\mu}\right)\right. \\
& \left.+i \sum_{a} \int d^{4} y X(y) \frac{\partial}{\partial y^{a}}\left[\Phi^{(a)}(y)\right]^{3}\right]
\end{aligned}
$$

where the right hand side not manifestly covariant and $\mathscr{N}$ is an unimportant normalization. Nonetheless the left hand side is manifestly covariant and frame independent. In contrast, if we had used the frame-covariant field redefinition Eq. (30), we would have found

$$
\int D \chi D V e^{i \int d^{4} x \chi(x) \partial_{\mu} V^{\mu}(x)} \propto \int D X D \Phi \exp \left[i \int d^{4} y M^{4} \ln \left(\operatorname{det}_{\mu a} \psi_{(a)}^{\mu}\right)+i \sum_{a} \int d^{4} y X(y) \frac{\partial}{\partial y^{a}} \Phi^{a}(y)\right]
$$

which is manifestly frame-covariant (i.e. manifestly Lorentz invariant if both $a$ and $\mu$ indices are transformed under Lorentz transformations). In this sense, the nonlinearity in the usage of the frame dependent variable causes greater "loss" of manifest covariance. Note the first term in the square bracket of Eq. (42) (which can be dropped in computations since it is a field independent term) is frame-covariant. That means in the frame-covariant field redefinitions, we can recover the manifest Lorentz invariance as long one moves to a Lorentz frame of $\psi_{(a)}^{\mu}=\delta^{\mu}{ }_{a}$ to eliminate $\psi$ and afterwards $a$ indices are transformed as Lorentz tensor indices in Lorentz transformations.

A similar loss of manifest covariance occurs in BTGT theory if one eliminates $\psi_{(a)}^{\mu}$ by a coordinate transformation and uses $\theta_{a}^{B}$ fields which carry the frame index $a$ treated as a scalar field label. On the other hand, because the theory is defined through a change of variables starting from the path integral measure $D A_{\mu}\left(\theta_{a}\right)$, the theory is covariant and frame independent. This change of variables was mainly motivated by the desire of rewriting the theory in terms of vierbeins and quantizing it through an unconstrained path integration over $\theta_{a}$. Because of the nonlinear field redefinition discussed in Eq. (41), frame-covariance does not really play a role in this argument 
(the field redefinition argument starting from $A_{\mu}$ ) for frame independence of the BTGT theory. On the other hand in Sec. 5 we discuss how frame covariance can enter the frame independence of a theory if we replace the crutch of using the field redefinition $A_{\mu}\left(\theta_{a}\right)$ with the symmetries of gauge invariance, BTGT invariance, $S_{4}$ symmetry, and a particular parity symmetry of the frame field.

\section{AN EXTENSION OF THE BTGT SYMMETRY}

The central object for BTGT in Eqs. (5) and (6) is the covariant tensor field $\left[G^{\mu v}(x)\right]^{i j}$. In this section, we explain which manifestly covariant functional degrees of freedom are thrown out when the BTGT theory is quantized in terms of $\theta_{a}^{A}$ and the consequences of this in comparing computations in different frames. In this way, we are able to identify an extension to the previously stated BTGT symmetry, the equivalence class of $\theta_{a}^{A}$ that give the same $A_{\mu}$. In particular, the extension is to include the invariance with respect to different frame choices $\left\{\psi_{(a)}^{\mu}, x[1]\right\}$ and was already implicit in previous papers [16-18].

We begin by giving a discussion of the Lorentz group representation properties of $\left[G^{\mu \nu}(x)\right]^{i j}$. With the aim of eventually accommodating Eqs. (4) and (5), start with a general set of complex numbers $\left[G^{\mu v}\right]^{i j}$ where the $\mu, v$ indices transform through the usual real matrices of the fundamental representation of $S O(1,3)$ and $i, j \in\{1, \ldots, N\} .{ }^{2}$ Because $\left[G^{\mu v}\right]^{i j}$ is required to be complex, for any fixed indices $i, j,\left[G^{\mu v}\right]^{i j}$ is a $16 \oplus 16$ representation of $S O(1,3)$ where the second 16 of the direct sum corresponds to the imaginary part of $\left[G^{\mu v}\right]^{i j}$. The object $\left[G^{\mu v}\right]^{i j}$ has $(2 \times 16) N^{2}$ number of real degrees of freedom.

In order to accommodate the choice of the exponentiation map Eq. (9) (motivated from the group representation property Eq. (4)), we impose the constraint

$$
\left(G^{\alpha \nu}\right)^{j i *}\left(G_{\alpha \mu}\right)^{j k}=\delta_{\mu}^{v} \delta^{i k}
$$

where the Latin indices such as $i, j$ refer to the space spanned by $i, j$ indices in the generator matrix $T_{i j}^{B}$ with $i, j \in\{1, \ldots, N\}$. One can easily show that this constraint corresponds to restricting $\left(G_{\mu}^{\lambda}\right)^{j k}$ to having $16 N^{2}$ real degrees of freedom. Hence, imposing this constraint reduced the number of degrees of freedom from $32 N^{2}$ to $16 N^{2}$. Indeed $\left(G^{\alpha}{ }_{\mu}\right)^{j k}$ can be thought of elements of $U(N, 3 N)$. Since Eq. (43) is a covariant constraint, manifest Lorentz invariance has been preserved.

\footnotetext{
${ }^{2}$ Here $N$ will eventually be identified with the gauge group generating matrix $\left(T^{A}\right)^{i j}$ being an $N \times N$ matrix as in Eq. (4).
} 
Since we want the smallest $\left(G^{\alpha}{ }_{\mu}\right)^{j k}$ representation to identify the smallest set of manifestly covariant degrees of freedom being thrown out when the BTGT theory is quantized in terms of $\theta_{a}$, we can then ask whether we can reduce the $16 N^{2}$ degrees of freedom further closer to $4 N^{2}$ without sacrificing covariance. To this end, note the raised Lorentz index matrix $\left(G^{\alpha \beta}\right)^{i j}$ in the ansatz Eq. (9) is symmetric in the Lorentz indices. We also know that any Lorentz transform orbits of $\left(G^{\alpha \beta}\right)^{i j}$ that is symmetric in the Lorentz indices remains symmetric. However, we cannot simply conclude from the usual $S O(1,3)$ decomposition $16=1 \oplus 3 \oplus 3 \oplus 9$ with the $10=1 \oplus 9$ being symmetric that a possible representation is $10 N^{2} \oplus 10 N^{2}$ since we need to satisfy Eq. (43). Indeed, we already know the restriction gives a smaller set since $16 N^{2}<20 N^{2}$. We can therefore try to solve Eq. (43) explicitly and impose the restriction of index symmetry.

Start by noting that a $U(1,3)$ group representation matrices are the set of matrices $U$ satisfying

$$
U^{\dagger} \eta U=\eta
$$

or equivalently

$$
U^{\alpha \nu *} U_{\alpha \mu}=\delta_{\mu}^{v}
$$

where $\eta$ is the Lorentz metric matrix reminiscent of Eq. (43). Hence, we will try to find $\left(G^{\alpha \beta}\right)^{i j}$ satisfying exchange symmetry in the Greek indices and Eq. (43) by starting with the $U(1,3)$ group matrix fundamental representation and then making each group matrix element be valued in a gauge Lie algebra matrix space in an appropriate way. As we will see below, these conditions will reduce the number of degrees of freedom from $16 N^{2}$ to $10 D(\mathscr{G})$ where $D(\mathscr{G}) \leq N^{2}$ is the dimension of the gauge group transforming according to Eq. (4).

The fundamental representation of $U(1,3)$ can be explicitly generated by the 16 matrices (ele- 
ments of the Lie algebra $u(1,3))$

$$
\begin{aligned}
& S \equiv\left\{\Xi^{1}, \Xi^{2}, \ldots, \Xi^{10}\right\} \equiv\left\{\left(\begin{array}{llll}
1 & 0 & 0 & 0 \\
0 & 0 & 0 & 0 \\
0 & 0 & 0 & 0 \\
0 & 0 & 0 & 0
\end{array}\right),\left(\begin{array}{cccc}
0 & 0 & 0 & 0 \\
0 & -1 & 0 & 0 \\
0 & 0 & 0 & 0 \\
0 & 0 & 0 & 0
\end{array}\right),\left(\begin{array}{cccc}
0 & 0 & 0 & 0 \\
0 & 0 & 0 & 0 \\
0 & 0 & -1 & 0 \\
0 & 0 & 0 & 0
\end{array}\right),\left(\begin{array}{cccc}
0 & 0 & 0 & 0 \\
0 & 0 & 0 & 0 \\
0 & 0 & 0 & 0 \\
0 & 0 & 0 & -1
\end{array}\right),\right. \\
& \left(\begin{array}{cccc}
0 & -1 & 0 & 0 \\
1 & 0 & 0 & 0 \\
0 & 0 & 0 & 0 \\
0 & 0 & 0 & 0
\end{array}\right),\left(\begin{array}{cccc}
0 & 0 & -1 & 0 \\
0 & 0 & 0 & 0 \\
1 & 0 & 0 & 0 \\
0 & 0 & 0 & 0
\end{array}\right),\left(\begin{array}{cccc}
0 & 0 & 0 & -1 \\
0 & 0 & 0 & 0 \\
0 & 0 & 0 & 0 \\
1 & 0 & 0 & 0
\end{array}\right),\left(\begin{array}{llll}
0 & 0 & 0 & 0 \\
0 & 0 & 1 & 0 \\
0 & 1 & 0 & 0 \\
0 & 0 & 0 & 0
\end{array}\right) \\
& \left.\left(\begin{array}{llll}
0 & 0 & 0 & 0 \\
0 & 0 & 0 & 1 \\
0 & 0 & 0 & 0 \\
0 & 1 & 0 & 0
\end{array}\right),\left(\begin{array}{llll}
0 & 0 & 0 & 0 \\
0 & 0 & 0 & 0 \\
0 & 0 & 0 & 1 \\
0 & 0 & 1 & 0
\end{array}\right)\right\}
\end{aligned}
$$

and

$$
\begin{aligned}
J \equiv\left\{\Xi^{11}, \Xi^{12}, \ldots, \Xi^{16}\right\} \equiv & \left\{i\left(\begin{array}{llll}
0 & 1 & 0 & 0 \\
1 & 0 & 0 & 0 \\
0 & 0 & 0 & 0 \\
0 & 0 & 0 & 0
\end{array}\right), i\left(\begin{array}{llll}
0 & 0 & 1 & 0 \\
0 & 0 & 0 & 0 \\
1 & 0 & 0 & 0 \\
0 & 0 & 0 & 0
\end{array}\right), i\left(\begin{array}{llll}
0 & 0 & 0 & 1 \\
0 & 0 & 0 & 0 \\
0 & 0 & 0 & 0 \\
1 & 0 & 0 & 0
\end{array}\right),\right. \\
& \left.i\left(\begin{array}{cccc}
0 & 0 & 0 & 0 \\
0 & 0 & -1 & 0 \\
0 & 1 & 0 & 0 \\
0 & 0 & 0 & 0
\end{array}\right), i\left(\begin{array}{llll}
0 & 0 & 0 & 0 \\
0 & 0 & 0 & 1 \\
0 & 0 & 0 & 0 \\
0 & -1 & 0 & 0
\end{array}\right), i\left(\begin{array}{llll}
0 & 0 & 0 & 0 \\
0 & 0 & 0 & 0 \\
0 & 0 & 0 & 1 \\
0 & 0 & -1 & 0
\end{array}\right)\right\}
\end{aligned}
$$

where the matrix entries correspond to Lorentz $\mu \nu$ entries. Although the matrices generated by $J$ form the group $S O(1,3)$ subgroup while the matrices generated by $S$ do not form a group, this does not mean we should restrict to the subgroup generated by $J$ for constructing BTGT because we are not looking for the group matrices but the representation basis tensors. ${ }^{3}$ Now, we impose the symmetry of $\left(G^{\alpha \beta}\right)^{i j}=\left(G^{\beta \alpha}\right)^{i j}$ (imposed by consistency with the ansatz Eq. (9)) as planned

\footnotetext{
${ }^{3} \mathrm{An} S U(2)$ group matrix can be represented as $\Gamma^{i j} \equiv \exp \left(i \theta^{A} \sigma^{A} / 2\right)^{i j}$ while the the basis tensor for this representation is $\psi^{i}$ with the orbit $\psi^{\prime}=\Gamma \psi$.
} 
by choosing $S$ to generate $\left(G_{\beta}^{\alpha}\right)^{i j}$. First, note

$$
K_{\beta}^{\alpha}=\exp \left[i \sum_{a=1}^{10} \varepsilon_{a}(x) \Xi^{a}\right]_{\beta}^{\alpha}
$$

gives a symmetric $K^{\alpha \beta}$. This property can be imported to describing $\left(G^{\alpha \beta}\right)^{j k}$. To solve Eq. (43), we still have to impose the condition on the Latin indices of $\left(G^{\alpha}{ }_{\mu}\right)^{j k}$. To see if the solution is possible, try

$$
\left(G_{\beta}^{\alpha}\right)^{i j}=\exp \left[i \sum_{a=1}^{10} \alpha_{a}(x) \Xi^{a}\right]_{\beta}^{\alpha}
$$

where $\alpha_{a}$ is Lie algebra valued: i. e.

$$
\alpha_{a} \equiv \sum_{C} \alpha_{a}^{C} T^{C}
$$

and the product $T^{C} \Xi^{a} T^{E} \Xi^{b}$ is defined as in the usual tensor product space:

$$
\left[\left(T^{C} \Xi^{a} T^{E} \Xi^{b}\right)_{\beta}^{\alpha}\right]^{i j}=\left(T^{C}\right)^{i k}\left(T^{E}\right)^{k j}\left(\Xi^{a}\right)_{\mu}^{\alpha}\left(\Xi^{b}\right)_{\beta}^{\mu} .
$$

It is easy to check that Eq. (43) can be satisfied as follows. First, expand:

$$
\begin{aligned}
\left(G^{\alpha \nu}\right)^{j i *}\left(G_{\alpha \mu}\right)^{j k} & =\sum_{\omega \alpha \lambda j}\left(G^{\alpha}{ }_{\omega} \eta^{\omega v}\right)^{j i *} \eta_{\alpha \lambda}\left(G^{\lambda}{ }_{\mu}\right)^{j k} \\
& =\sum_{j} \eta\left(\exp \left[-i \sum_{a=1}^{10} \alpha_{a}(x) \Xi^{a \dagger}\right]\right)^{i j} \eta\left(\exp \left[i \sum_{a=1}^{10} \alpha_{a}(x) \Xi^{a}\right]\right)^{j k} .
\end{aligned}
$$

Using the property $\eta \Xi^{a \dagger} \eta=\Xi^{a}$, we conclude this is equivalent to Eq. (43).

Even though $T^{C} \Xi^{a}$ is not in general a group generator, we are most concerned with whether the orbit of $\left(G_{\beta}^{\alpha}\right)^{i j}$ under Lorentz transformations and can be written in the form of Eq. (49). Note that there generically exists a solution $\tilde{\alpha}_{a}^{A}(x)$ to

$$
\left(\exp \left[i \sum_{a=1}^{10} \sum_{A} \alpha_{a}^{A}(x) T^{A} \Xi^{a}\right]_{\beta}^{\alpha}\right)^{i j} \exp \left[i \sum_{B} \Theta^{B}(x) T^{B}\right]^{j k}=\left(\exp \left[i \sum_{a=1}^{10} \sum_{A} \tilde{\alpha}_{a}^{A}(x) T^{A} \Xi^{a}\right]_{\beta}^{\alpha}\right)^{i k}
$$

since

$$
\left[T^{A} \Xi^{a}, T^{B} \mathbb{I}\right]=i f^{A B C} T^{C} \Xi^{a} .
$$

Furthermore, since

$$
\exp \left[i \sum_{a=1}^{10} \sum_{A} \alpha_{a}^{A}(x) T^{A} \Xi^{a}\right]^{\alpha \beta}=\exp \left[i \sum_{a=1}^{10} \sum_{A} \alpha_{a}^{A}(x) T^{A} \Xi^{a}\right]^{\beta \alpha}
$$


(with the $T^{A}$ associated Latin indices suppressed) and the Lorentz transform induced orbits preserve this symmetry, one generically expects there to be a solution to

$$
\Lambda_{\alpha}^{\mu} \Lambda_{\beta}^{v} \exp \left[i \sum_{a=1}^{10} \sum_{A} \alpha_{a}^{A}(x) T^{A} \Xi^{a}\right]^{\alpha \beta}=\exp \left[i \sum_{a=1}^{10} \sum_{B} \bar{\alpha}_{a}^{B}(\Lambda x) T^{B} \Xi^{a}\right]^{\mu \nu}
$$

as we will now verify.

For verification, we give explicit representation of the covariant $10 \times D(A)$ degrees of freedom in $\alpha_{a}^{A}(x) T^{A} \Xi^{a}$, which can be given in many forms. One form is

$$
K_{v}^{\mu}(x)=\exp \left[i \sum_{a=1}^{10} \alpha_{a}(x) \Xi^{a}\right]
$$

which under Lorentz transformation transforms as

$$
\begin{gathered}
\bar{\alpha}_{c}(\bar{x})=\frac{1}{N_{c}} \sum_{a} \alpha_{a}\left(\Lambda^{-1} \bar{x}\right) \operatorname{Tr}\left(\Xi^{c} \Lambda \Xi^{a} \Lambda^{-1}\right) \\
\operatorname{Tr}\left(\Xi^{c} \Xi^{f}\right)=\delta^{c f} N_{c}
\end{gathered}
$$

where $\alpha_{a}$ is Lie algebra valued: i. e.

$$
\alpha_{a} \equiv \sum_{C} \alpha_{a}^{C} T^{C}
$$

where if $\xi_{(f)}^{* l}\left(K_{v}^{\mu}\right)^{l m}$ is to transform as an antifundamental, we can set $T^{C}$ equal to the negative of the Hermitian conjugate of the fundamental representation matrices and sum over $D(A)$ of them. Note also that the trace here is only over the Lorentz spacetime indices. This form is useful in counting the degrees of freedom and its concise relationship to the generators of $u(1,3)$. A more familiar Lorentz tensorial form is given by making a variable change $\alpha_{a} \rightarrow \alpha^{\alpha \beta}=\alpha^{(\alpha \beta)}$ where

$$
\begin{gathered}
\alpha_{a}(x)\left(\Xi^{a}\right)^{\mu \nu}=\frac{1}{2} \alpha^{\alpha \beta}\left(\Xi_{\alpha \beta}\right)_{\lambda}^{\mu} \eta^{\lambda v} \\
\alpha_{a}(x)\left(\Xi^{a}\right)^{\mu}{ }_{\lambda} \eta^{\lambda v}=\alpha^{\alpha \beta} \delta_{(\alpha}^{\mu}{ }_{\left({ }^{\nu}\right)}
\end{gathered}
$$


and

$$
\begin{aligned}
& \left\{\Xi^{00} \eta, \Xi^{11} \eta, \Xi^{22} \eta, \Xi^{33} \eta\right\} \equiv\left\{2\left(\begin{array}{cccc}
1 & 0 & 0 & 0 \\
0 & 0 & 0 & 0 \\
0 & 0 & 0 & 0 \\
0 & 0 & 0 & 0
\end{array}\right), 2\left(\begin{array}{cccc}
0 & 0 & 0 & 0 \\
0 & -1 & 0 & 0 \\
0 & 0 & 0 & 0 \\
0 & 0 & 0 & 0
\end{array}\right), 2\left(\begin{array}{cccc}
0 & 0 & 0 & 0 \\
0 & 0 & 0 & 0 \\
0 & 0 & -1 & 0 \\
0 & 0 & 0 & 0
\end{array}\right),\right. \\
& \left.2\left(\begin{array}{cccc}
0 & 0 & 0 & 0 \\
0 & 0 & 0 & 0 \\
0 & 0 & 0 & 0 \\
0 & 0 & 0 & -1
\end{array}\right)\right\} \\
& \left\{\Xi^{01} \eta, \Xi^{10} \eta, \Xi^{02} \eta, \Xi^{20} \eta, . .\right\} \equiv\left\{\left(\begin{array}{cccc}
0 & 1 & 0 & 0 \\
0 & 0 & 0 & 0 \\
0 & 0 & 0 & 0 \\
0 & 0 & 0 & 0
\end{array}\right),\left(\begin{array}{llll}
0 & 0 & 0 & 0 \\
1 & 0 & 0 & 0 \\
0 & 0 & 0 & 0 \\
0 & 0 & 0 & 0
\end{array}\right),\left(\begin{array}{llll}
0 & 0 & 1 & 0 \\
0 & 0 & 0 & 0 \\
0 & 0 & 0 & 0 \\
0 & 0 & 0 & 0
\end{array}\right),\right. \\
& \left.\left(\begin{array}{llll}
0 & 0 & 0 & 0 \\
0 & 0 & 0 & 0 \\
1 & 0 & 0 & 0 \\
0 & 0 & 0 & 0
\end{array}\right), \cdots\right\}
\end{aligned}
$$

With this definition, the fields $\alpha^{\mu v}$ have the following Lorentz transformation property:

$$
\alpha^{\kappa \phi}\left(\Lambda^{-1} \bar{x}\right) \Lambda^{\mu}{ }_{\kappa} \Lambda_{\phi}^{v}=\bar{\alpha}^{\mu v}(\bar{x})
$$

which is obviously useful for constructing field theories. From this, it is clear that one can recover a manifestly frame independent description with $10 \times D(\mathscr{G})$ degrees of freedom whereas the diagonal fields $\left\{\alpha^{00}, \alpha^{11}, \alpha^{22}, \alpha^{33}\right\}$ are the $\theta_{a}$ degrees of freedom.

Since $S$ has 10 elements, we see that we can describe a manifestly frame independent covariant complex tensor $\left(G_{\mu}^{\alpha}\right)^{j k}$ with $10 \times D(\mathscr{G})$ real degrees of freedom. The subset of $S$ given by $\left\{\Xi_{1}, \Xi_{2}, \Xi_{3}, \Xi_{4}\right\}$ (the Cartan subalgebra of $u(1,3)$ ) generate the Abelian group $U(1)^{4}$ as usual, and it is this group manifold that is parameterized by the BTGT fields $\theta_{a}^{B}$ for any fixed $B$. Hence, we have arrived at our answer: it is the $6 D(\mathscr{G})$ related to the non-Cartan-subalgebra elements of $u(1,3)$ in $S$ that are lost in using the $\theta_{a}^{B}$ ansatz Eq. (9) and leads to a frame-dependent description of a frame independent theory.

One consequence of describing the covariant frame independent theory using only $4 \times D(\mathscr{G})$ degrees of freedom (instead of $10 \times D(\mathscr{G})$ ) is that when one compares $G^{\mu v}$ computations executed 
in two different $x[1]$ frame choices (see Eq. (24) for the definition of $x[1]$ ), one is actually considering different tensors and not the components of the same tensor in different Lorentz frames. To be more precise, suppose one $x[1]$ choice (with coordinate basis $e_{v}$ ) is related to the other $\underline{x}[1]$ choice through a Lorentz transform $\underline{x}[1]=x[2]=\Lambda x[1]$. Suppose the field $G^{\mu v}$ in $x[1]$ coordinates is computed with a basis choice $\psi_{(a)}^{\mu}$ :

$$
G^{\mu}{ }_{v}(x[1]) e_{\mu} \otimes e^{v}=\left(\exp \left[-i \sum_{a} \theta_{a}(x[1]) \psi_{(a)} \psi_{(a)} \eta^{a a}\right]\right)_{v}^{\mu} e_{\mu} \otimes e^{v}
$$

In $x[2]=\Lambda x[1]$ coordinates where $\bar{\psi}_{(a)}^{\mu}=\Lambda_{\lambda}^{\mu} \psi_{(a)}^{\lambda}$ and $\bar{e}_{\mu}=\left(\Lambda^{-1}\right)_{\mu}^{\beta} e_{\beta}$, we know

$$
G^{\mu}{ }_{v}(x[1]) e_{\mu} \otimes e^{v}=\bar{G}^{\mu}{ }_{v}(x[2]) \bar{e}_{\mu} \otimes \bar{e}^{v}=\left(\exp \left[-i \sum_{a} \theta_{a}\left(\Lambda^{-1} x[2]\right) \bar{\psi}_{(a)} \bar{\psi}_{(a)} \eta^{a a}\right]\right)_{v}^{\mu} \bar{e}_{\mu} \otimes \bar{e}^{v}
$$

where we have used the scalar transformation property of $\theta_{a}$. Now, suppose one considers the same choice of basis $\psi_{(a)}^{\mu}$ in $\underline{x}[1]=x[2]$ frame and executes the computation. One can easily obtain

$$
\underline{G}^{\mu}{ }_{v}(x[2]) \bar{e}_{\mu} \otimes \bar{e}^{v}=\left(\exp \left[-i \sum_{a} \Theta_{a}(x[2]) \psi_{(a)} \psi_{(a)} \eta^{a a}\right]\right)_{v}^{\mu} \bar{e}_{\mu} \otimes \bar{e}^{v}
$$

where

$$
\sum_{a} \Theta_{a}(x[2]) \psi_{(a)}^{\alpha} \psi_{(a)}^{\beta} \eta^{a a} \neq \sum_{a} \theta_{a}\left(\Lambda^{-1} x[2]\right) \bar{\psi}_{(a)}^{\alpha} \bar{\psi}_{(a)}^{\beta} \eta^{a a}
$$

This means

$$
\underline{G}^{\mu}{ }_{v}(x[2]) \bar{e}_{\mu} \otimes \bar{e}^{v} \neq \bar{G}^{\mu}{ }_{v}(x[2]) \bar{e}_{\mu} \otimes \bar{e}^{v}
$$

even though

$$
\left[\underline{G}^{-1 \alpha \beta}(x[2])\right]\left[\frac{\partial}{\partial x^{\alpha}[2]} \underline{G}_{\beta \mu}(x[2])\right] \bar{e}^{\mu}=\left[\bar{G}^{-1 \alpha \beta}(x[2])\right]\left[\frac{\partial}{\partial x^{\alpha}[2]} \bar{G}_{\beta \mu}(x[2])\right] \bar{e}^{\mu} .
$$

Consequently, the map of $G_{\beta}^{\alpha}$ to $A_{\mu}$ together with the covariance of $G^{\alpha}{ }_{\beta}$ and $A_{\mu}$ induce an equivalence class of of tensors $G^{\mu v} e_{\mu} \otimes e_{v} \sim \underline{G}^{\alpha \beta} \bar{e}_{\alpha} \otimes \bar{e}_{\beta}$ even though $G^{\mu v} e_{\mu} \otimes e_{v}=\bar{G}^{\mu v} \bar{e}_{\mu} \otimes$ $\bar{e}_{\nu} \neq \underline{G}^{\alpha \beta} \bar{e}_{\alpha} \otimes \bar{e}_{\beta}$

Although we already know that BTGT transform is identified as an $A_{\mu}$ equivalence class, what we learn from this section is that the equivalence class is a combination of an $\left\{\psi_{(a)}^{\mu}, x[1]\right\}$ choice in addition to the BTGT transform. That is because the BTGT symmetry transformations (see Eq. (16)) defining a set of equivalence class conditions involve fewer than $4 \times D(\mathscr{G})$ real functional degrees of freedom since according to the constraint Eq. (17), the equivalence class functions in the BTGT are functions of one fewer dimension (i.e. 3-dimensional functions in a 4-dimensional 
spacetime) which is certainly fewer than the $6 \times D(\mathscr{G})$ degrees of freedom required to restore manifest frame independence. The data of $\left\{\psi_{(a)}^{\mu}, x[1]\right\}$ chooses which $4 \times D(\mathscr{G})$ of the $10 \times D(\mathscr{G})$ real functional degrees of freedom that one is calling $\theta_{a}^{B}$. BTGT is automatically invariant under $\left\{\psi_{(a)}^{\mu}, x[1]\right\}$ choice since the path integral is defined originally with respect to $A_{\mu}$.

One might wonder whether working with $\theta_{a}$ frame dependent fields will require a strong frame dependence of the counter terms. In our previous explicit computations [17, 18], we saw no evidence for the variable change playing a role at one-loop. As we will see in the next section, there is a good reason why this change of variables does not lead to disastrous loss of frame invariance through the counter terms: the combination of BTGT, gauge, and Lorentz invariance, together with $S_{4}$ symmetry and a parity symmetry of the frame field-implicit in the BTGT formulation leads to manifest frame independence of the action.

\section{FRAME INDEPENDENCE WITHOUT RELYING ON THE $A_{\mu}$ FIELD}

Thus far, we have focused on the frame independence of BTGT being a consequence of a field redefinition of the manifestly covariant field $A_{\mu}\left(\theta_{a}\right)$. We know that $A_{\mu}$ is required for gauge invariance. Furthermore, the relationship between $\theta_{a}$ and $A_{\mu}$ is constrained by BTGT invariance, gauge invariance, and the choice of $\left\{\psi_{(a)}^{\mu}, x[1]\right\}$. In this section, we show how to remove the crutch of $A_{\mu}$ field (at the action level) with two additional conditions to gauge and BTGT invariance. More explicitly, we show how to construct theories with $\left\{\psi_{(a)}^{\mu}, x[1]\right\}$ choices that do not have any physical effect on the theory (except possibly for zero modes) if we add the following conditions to the theory: an $S_{4}$ permutation symmetry generated by exchanges

$$
\left\{\eta^{a a}, \psi_{(a)}, U_{a}\right\} \leftrightarrow\left\{\eta^{b b}, \psi_{(b)}, U_{b}\right\}
$$

for any BTGT indices $a$ and $b$ in $\{0, \ldots, 3\}$, and a rigid rescaling symmetry

$$
\psi_{(a)}^{\mu} \rightarrow \lambda_{a} \psi_{(a)}^{\mu}
$$

of the basis fields for any constant $\lambda_{a} \neq 0$. This construction is done without starting from the theory constructed from gauge connection $A_{\mu}$.

First, we begin with some comments about conventions chosen. Via the rescaling symmetry of Eq. (74), one can normalize the orthogonal basis set $\psi_{(a)}^{\mu}$ such that

$$
\left(\psi_{(a)}^{\lambda} \psi_{(a) \lambda}\right)^{-1}=\eta^{a a}
$$


without loss of generality. After normalizing, there remains a residual $\psi_{(a)}^{\mu}$ parity symmetry

$$
\psi_{(a)}^{\mu} \rightarrow \operatorname{sign}\left(\lambda_{a}\right) \psi_{(a)}^{\mu}
$$

that corresponds to the $P T$ discrete subgroup of the $O(1,3)$ Lorentz group. The choice of Eq. (75) is implied for the rest of this section. In addition, although $\theta_{a}$ and $U_{a}=e^{i \theta_{a}}$ are functionally equivalent, the advantage of $U_{a}$ is that the symmetry transformations are tensorial (by construction of BTGT), while the non-Abelian $\theta_{a}$ transforms inhomogeneously and nonlinearly. Constructing BTGT and gauge invariants is therefore simpler when working with $U_{a}$.

The proposition we would like to establish in this section is the following. Suppose the BTGT and gauge invariant action $S\left[\psi_{(a)}^{\mu}, U_{a}, \phi\right]$ is invariant under the symmetries of Eqs. (73) and (74), where $\phi$ is a scalar matter field transforming under the fundamental representation of gauge group $\mathscr{G}$. Let the action $S$ be derived from a local Lagrangian $\mathscr{L}$ and renormalizable. Given these conditions, the action is frame independent as defined in Appendix A Explicitly, there exists a change of variables $V^{\mu}\left(\psi_{(a)}^{\mu}, U_{a}\right)$ and a new action $\tilde{S}\left[V^{\mu}, \phi\right]$ that is independent of $\psi_{(a)}^{\mu}$ such that

$$
S\left[\psi_{(a)}^{\mu}, U_{a}, \phi\right]=\tilde{S}\left[V^{\mu}, \phi\right] .
$$

The intuition is that gauge invariance, BTGT invariance, and the conditions of Eqs. (73) and (74) combine to restrict the theory to only being constructed from gauge covariant derivatives of the form

$$
D^{\mu}(\cdot)=\sum_{a} \eta^{a a} \psi_{(a)}^{\mu} \psi_{(a)}^{\lambda} U_{a} \partial_{\lambda}\left(U_{a}^{-1} \cdot\right)
$$

where the argument $\cdot$ stands for any field in some representation $R$ of the gauge group and $U_{a}=$ $e^{i \theta_{a}^{A} T_{(R)}^{A}}$, where $T_{(R)}^{A}$ are the basis elements of the Lie algebra in representation $R$. Eq. (78) has a decomposition into $\partial^{\mu}$ and vector field

$$
V^{\mu}\left(\psi_{(a)}^{\mu}, U_{a}\right)=i \sum_{a} \eta^{a a} \psi_{(a)}^{\mu} \psi_{(a)}^{\lambda} U_{a} \partial_{\lambda}\left(U_{a}^{-1}\right)
$$

Due to gauge invariance, the Lagrangian must be composed from covariant derivatives of Eq. (78). The only $U_{a}$ dependence of the action $S\left[\psi_{(a)}^{\mu}, U_{a}, \phi\right]$ is then of the form Eq. (79). Therefore we end up with an action $\tilde{S}\left[\psi_{(a)}^{\mu}, V^{\mu}\left(\psi_{(a)}^{\mu}, U_{a}\right), \phi\right]$ that satisfies Eq. (77). The residual $\psi_{(a)}^{\mu}$ frame dependence of $\tilde{S}$ is finally removed by restricting to renormalizable terms consistent with the parity and $S_{4}$ symmetry properties given in Eqs. (76) and (73).

Let us now proceed with the details. In Appendix B, it is shown that the most general BTGT invariant Lagrangian must be a function of BTGT invariant monomials $U_{a} \partial_{(a)} U_{a}^{-1}$ and $\partial_{\mu}\left(U_{a} \partial_{(a)} U_{a}^{-1}\right)$. Explicitly, the functional dependence of the Lagrangian is 


$$
\mathscr{L}=\mathscr{L}\left(\psi_{(a)}^{\mu}, U_{a} \partial_{(a)} U_{a}^{-1}, \partial_{\mu}\left(U_{a} \partial_{(a)} U_{a}^{-1}\right), \phi, \partial_{\mu} \phi, \phi^{\dagger}, \partial_{\mu} \phi^{\dagger}\right),
$$

where $\partial_{(a)} U_{a}^{-1} \equiv \psi_{(a)}^{\mu} \partial_{\mu} U_{a}^{-1}$ is the projected derivative. We next use gauge invariance to further restrict the theory. The gauge covariant derivative which has the usual transformation properties is

$$
D^{\mu}(\cdot)=\sum_{a, b} c^{a b} \psi_{(a)}^{\mu} U_{b} \partial_{(b)}\left(U_{b}^{-1} \cdot\right)
$$

where any choice of the constant $c^{a b}$ is compatible with both gauge and BTGT invariance. Imposing the conditions of Eqs. (73) and (76) restricts this to

$$
c^{a b}=\delta^{a b} \eta^{a a}=\eta^{a b}
$$

which corresponds to the covariant derivative of Eq. (78). Given Eq. (82), the projected covariant derivative of $U_{a}$ is zero: $D_{(a)} U_{a}=\psi_{(a) \mu} D^{\mu} U_{a}=U_{a} \partial_{(a)}\left(U_{a}^{-1} U_{a}\right)=0$. Any term containing such a derivative is therefore zero after imposing gauge invariance by promoting ordinary derivatives to covariant derivatives defined by Eq. (78). Since BTGT invariance requires projected derivatives $\partial_{(a)} U_{a}^{-1}$, all terms involving projected derivatives of $U_{a}$ go to zero once the theory is made gauge covariant. The terms consistent with gauge invariance are then composed of $F^{\mu v} \equiv i\left[D^{\mu}, D^{v}\right]$, $D^{\mu} \phi$, and its conjugate $D^{\mu} \phi^{\dagger}$. Explicitly terms of fields,

$$
\begin{aligned}
F^{\mu \nu} & =i \sum_{a, b} \eta^{a a} \eta^{b b} \psi_{(a)}^{[\mu} \psi_{(b)}^{v]} U_{a} \partial_{(a)}\left(U_{a}^{-1} U_{b} \partial_{(b)}\left(U_{b}^{-1}\right)\right) \\
& =\partial^{[\mu} V^{v]}\left(\psi_{(a)}^{\mu}, U_{a}\right)-i V^{[\mu}\left(\psi_{(a)}^{\mu}, U_{a}\right) V^{v]}\left(\psi_{(a)}^{\mu}, U_{a}\right),
\end{aligned}
$$

and

$$
\begin{aligned}
D^{\mu} \phi & =\sum_{a} \eta^{a a} \psi_{(a)}^{\mu} U_{a} \partial_{(a)}\left(U_{a}^{-1} \phi\right) \\
& =\partial^{\mu} \phi-i V^{\mu}\left(\psi_{(a)}^{\mu}, U_{a}\right) \phi
\end{aligned}
$$

where $V^{\mu}(\psi, U)$ is defined in Eq. (79). It is interesting that the commutator of fields does not appear in the BTGT formalism Eq. (83). More precisely, the gauge curvature tensor $F^{\mu v}$ is homogeneous in ordinary derivatives, just like in the Abelian theory. We defer the exploration of this property to a future work.

The $\psi_{(a)}^{\mu}$ parity symmetry of Eq. (76) only allows $\psi_{(a)}^{\mu}$ to appear in the bilinear invariant $\left(H^{a}\right)^{\mu v}$ of Eq. (10). The most general BTGT and gauge invariant renormalizable Lagrangian consistent 
with the $\psi_{(a)}^{\mu}$ parity symmetry of Eq. (76) is

$$
\mathscr{L}=\mathscr{L}_{Y M}+\mathscr{L}_{C P}+\mathscr{L}_{\text {matter }}
$$

where

$$
\begin{gathered}
\mathscr{L}_{Y M}=\sum_{a, b} x_{a b}\left(H^{a}\right)^{\mu v}\left(H^{b}\right)^{\rho \lambda} \operatorname{Tr}\left(F_{\mu \rho} F_{v \lambda}\right), \\
\mathscr{L}_{C P}=\sum_{a, b} y_{a b}\left(H^{a}\right)^{\mu v}\left(H^{b}\right)^{\rho \lambda} \operatorname{Tr}\left(F_{\mu \rho} \tilde{F}_{v \lambda}\right), \\
\mathscr{L}_{\text {matter }}=\sum_{a} z_{a}\left(H^{a}\right)^{\mu v} D_{\mu} \phi^{\dagger} D_{\nu} \phi-V_{\phi}\left(\phi, \phi^{\dagger}\right),
\end{gathered}
$$

for some constants $x_{a b}, y_{a b}$, and $z_{a}, \tilde{F}_{\alpha \beta} \equiv \frac{1}{2} \varepsilon_{\alpha \beta \mu v} F^{\mu v}$ is the dual field strength tensor, and $V_{\phi}\left(\phi, \phi^{\dagger}\right)$ is a gauge invariant potential. The residual basis dependence of $\mathscr{L}$ is removed by applying the permutation symmetry Eq. (73); this leads to the restriction

$$
x_{a b}=\left\{\begin{array}{ll}
x+x^{\prime} & a=b \\
x & a \neq b
\end{array} \quad y_{a b}=\left\{\begin{array}{ll}
y+y^{\prime} & a=b \\
y & a \neq b
\end{array} \quad z_{a}=z\right.\right.
$$

for some constants $x, x^{\prime}, y, y^{\prime}$, and $z$. The Lagrangian then reduces to

$$
\begin{aligned}
\mathscr{L}= & x \sum_{a, b}\left(H^{a}\right)^{\mu v}\left(H^{b}\right)^{\rho \lambda} \operatorname{Tr}\left(F_{\mu \rho} F_{v \lambda}\right)+x^{\prime} \sum_{a}\left(H^{a}\right)^{\mu v}\left(H^{a}\right)^{\rho \lambda} \operatorname{Tr}\left(F_{\mu \rho} F_{v \lambda}\right) \\
& +y \sum_{a, b}\left(H^{a}\right)^{\mu v}\left(H^{b}\right)^{\rho \lambda} \operatorname{Tr}\left(F_{\mu \rho} \tilde{F}_{v \lambda}\right)+y^{\prime} \sum_{a}\left(H^{a}\right)^{\mu v}\left(H^{a}\right)^{\rho \lambda} \operatorname{Tr}\left(F_{\mu \rho} \tilde{F}_{v \lambda}\right) \\
& +z \sum_{a}\left(H^{a}\right)^{\mu v} D_{\mu} \phi^{\dagger} D_{v} \phi-V_{\phi}\left(\phi, \phi^{\dagger}\right) \\
= & x \operatorname{Tr}\left(F_{\mu \rho} F^{\mu \rho}\right)+y \operatorname{Tr}\left(F_{\mu \rho} \tilde{F}^{\mu \rho}\right)+z D^{\mu} \phi^{\dagger} D_{\mu} \phi-V_{\phi}\left(\phi, \phi^{\dagger}\right)
\end{aligned}
$$

which is frame independent. The frame dependent $x^{\prime}$ and $y^{\prime}$ terms are zero because $F_{\mu \nu}$ and its dual are anti-symmetric while the coefficients satisfy the identity- $\left(H^{a}\right)^{\mu \nu}\left(H^{a}\right)^{\rho \lambda}=\left(H^{a}\right)^{\mu \rho}\left(H^{a}\right)^{v \lambda}$. Eq. (93) with $x=-\frac{1}{4 g^{2}}, y=-\frac{\theta_{Q C D}}{32 \pi^{2}}$ and $z=1$ corresponds to the usual Yang-Mills plus matter Lagrangian. We see from Eq. (93) that the only $\psi_{(a)}^{\mu}$ dependence of $S\left[\psi_{(a)}^{\mu}, U_{a}, \phi\right]$ occurs in $D^{\mu} \phi$ and $F^{\mu v}$, and from Eq. (84) and Eq. (86) we see that this $\psi_{(a)}^{\mu}$ dependence only occurs in form of Eq. (79). Therefore the action $S\left[\psi_{(a)}^{\mu}, U_{a}, \phi\right]$ can written as Eq. (77), where the action $\tilde{S}\left[V^{\mu}, \phi\right]$ is the usual non-Abelian gauge theory action in terms of gauge connection $V^{\mu}$. Since $\tilde{S}$ contains no $\psi_{(a)}^{\mu}$ dependence, the BTGT action $S\left[\psi_{(a)}^{\mu}, U_{a}, \phi\right]$ is thus frame independent.

One possible limitation that prevents an extension of frame independence to the quantum theory itself is the path integral measure. Consider a change of variables of integration in the partition 
function from $\theta_{a}$ to $V_{\mu}$ defined in Eq. (79). The change of variables affects the theory via the measure change by

$$
D \theta=\left|\frac{\delta \theta_{a}}{\delta V_{\mu}}\right| D V=\mathscr{J}_{\psi} D V
$$

where the subscript on the Jacobian $J_{\psi}$ indicates that the theory may still be frame dependent. For instance, in the Abelian case we write the Jacobian inverse as

$$
\mathscr{J}_{\psi}^{-1}=\operatorname{det}_{\mu a}\left(\eta^{a a} \psi_{(a)}^{\mu} \psi_{(a)}^{\lambda} \partial_{\lambda}\right)=\operatorname{det}_{\mu a}\left(\left(H^{a}\right)_{\lambda}^{\mu} \partial^{\lambda}\right)
$$

and we see that explicit frame dependence coming from the zero modes of derivative operator $\left(H^{a}\right)_{\lambda}^{\mu} \partial^{\lambda}$. Nonetheless, this Jacobian should be frame independent apart from the zero modes, which do not affect perturbation theory.- This is consistent with the explicit one loop computations done in both the Abelian [17] and non-Abelian [18] cases. The residual $\psi_{(a)}^{\mu}$ dependence of the theory might also be removable-by averaging over all $\psi_{(a)}^{\mu}$, i.e. taking a path integral over all possible $\psi_{(a)}^{\mu}$. Another possible (but even less likely) obstruction to the theory being frame independent would be the non-invariance of the measure under the symmetries such as the $S_{4}$ permutation and parity symmetries. We will not address these issues further in this paper.

There are also higher mass dimension non-renormalizable terms with $\psi_{(a)}^{\mu}$ frame dependence that can be written down in the Lagrangian- consistent with all the symmetries. . For example, terms such as

$$
\mathscr{L}_{\text {f.dep. } 1}=\sum_{a}\left(H^{a}\right)^{\mu v}\left(H^{a}\right)^{\rho \sigma} D_{\mu} \phi^{\dagger} D_{v} \phi D_{\rho} \phi^{\dagger} D_{\sigma} \phi \operatorname{Tr}\left(F^{2}\right)
$$

and

$$
\mathscr{L}_{\text {f.dep } .2}=\sum_{a}\left(H^{a}\right)^{\mu v}\left(H^{a}\right)^{\rho \sigma}\left(H^{a}\right)^{\alpha \beta}\left(H^{a}\right)^{\gamma \delta} F_{\mu \alpha} F_{v \beta} F_{\rho \gamma} F_{\sigma \delta}
$$

belong in this category of operators. Even though they are not eliminated by pure gauge basis invariance, they are forbidden by a scaling symmetry of $H_{a} \rightarrow e^{\phi_{a}} H_{a}, U_{a} \rightarrow e^{-\phi_{a}} U_{a}, U_{a}^{\dagger} \rightarrow e^{-\phi_{a}} U_{a}^{\dagger}$ for real global parameter $\phi_{a}$. Dividing by $\operatorname{Tr}\left[H_{a}\right]^{2}$ in the sum of Eq. (96) would yield an invariant, but these terms will not arise in the effective action as as counter-terms do not involve inverse powers of fields. We have thus far ignored the measure issue under this scaling, but we suspect that it should not be an issue outside of zero modes. This is discussed further-in Appendix C, 


\section{SUMMARY}

In this paper, we have clarified the representational aspects of the BTGT quantization variable $\theta_{a}^{A} H_{\mu \nu}^{a}$ which contains the information about the gauge vierbein analog variable $\left[G_{(f) \beta}^{\alpha}(x)\right]^{i}$.

Our first result was to show that $\left(H^{a}\right)^{\mu v}$ when written in terms of $\psi_{(a)}^{\mu}$ in Eq. (10) can be understood as a tensor made from a bilinear combination of Jacobian factors (Eq. (25)) in going from an arbitrary chosen inertial frame $x[1]$ to another inertial frame. This means $\theta_{a}^{A}$ are frame $x[1]$ choice dependent fields. Afterwards, we showed through Eq. (49) how a manifestly covariant description of $\left[G_{(f) \beta}^{\alpha}(x)\right]^{i}$ without reference to $x[1]$ can be accomplished through $\left(G^{\alpha \beta}\right)^{i j}$ which has $10 D(\mathscr{G})$ degrees of freedom (where $D(\mathscr{G})$ is the dimension of the gauge group transforming according to Eq. (4)). This means by quantizing using $\theta_{a}^{A}$, we are setting $6 D(\mathscr{G})$ degrees of freedom to effectively to zero.

Despite this arbitrariness, because BTGT was defined through a field redefinition from $A_{\mu}^{B}$ to $\theta_{a}^{B}$ in [16-18], the BTGT formalism is manifestly Lorentz invariant and $x[1]$ choice independent. As a corollary, we have shown through Eqs. (71) and (72) that different $x[1]$ choices leads to an equivalence class of $\theta_{a}^{A}$. This equivalence class is distinct from the BTGT and gauge symmetry, and it is inherent in the usage of $\theta_{a}^{A}$ for quantization. Although this last statement is in some sense trivial since the equivalence arises from merely a field redefinition, computations at one loop [16-18] did not show a sensitivity to the path integral variable change Jacobian. This makes this equivalence class statement less trivial.

We then partially explained why the $x[1]$ frame dependence is disappearing in [16-18] by showing explicitly in Sec. 5 that BTGT invariance, gauge invariance, renormalizablity, and a couple of discrete symmetries associated with the frame-dependent variables $\left(S_{4}\right.$ permutation symmetry and a parity symmetry related to $P T$ symmetry) make the action $x[1]$ choice independent even when the action depends on both $\psi_{(a)}^{\mu}$ and $U_{a}=\exp \left[i \theta_{a}^{A} T^{A}\right]$ (both of which are $x[1]$ dependent). The full quantum generating functional is almost $x[1]$ independent except for the issues associated with the zero modes of the Jacobian associated with the path integration measure chage. Hence, the perturbative computation is also argued to be $x[1]$ independent.

The work presented in this paper has several obvious extensions. First, although we have focused in Sec. 5] on renormalizable BTGT theories, the frame independence may be generalizable to nonrenormalizable theories through extended symmetries of the form in Eq. (C1). Secondly, we have explicitly constructed the the frame independence in Sec. 5 including only gauged scalar 
matter fields. It would be interesting to extend this to higher spin fields. Thirdly, we noted in Eq. (83) that the non-Abelian field strength tensor in the BTGT formalism is homogeneous in the derivatives just as in the Abelian theory. It would be interesting to use this property as well as the property $D_{(a)} U_{a}=0$ for constructing novel semiclassical solutions.

Perhaps the most interesting extension is to embed Eq. (49) into coset model of gauge fields. (For other related efforts in this direction, see e.g. [19-21].) For this effort, it would be useful to rewrite the BTGT symmetry in terms of $G_{\mu \nu}$ only without referring to the $U_{a}$ field. Such coset constructions will naively generate $6 D(\mathscr{G})$ additional degrees of freedom than what is observed. It would be interesting whether these additional degrees of freedom can be sufficiently hidden for phenomenological consistency.

\section{Acknowledgments}

This work was supported in part by the DOE through grant DE-SC0017647.

\section{Appendix A: Definitions}

In this section, we explicitly define some of the terms used in the paper.

\section{Basis fields $\psi_{(a)}^{\mu}$}

The basis fields $\psi_{(a)}^{\mu}$ are four constant/global fields $\left\{\psi_{(0)}^{\mu}, \psi_{(1)}^{\mu}, \psi_{(2)}^{\mu}, \psi_{(3)}^{\mu}\right\}$ that form an orthogonal basis of spacetime that corresponds to a particular rest frame $x[1]$ :

$$
\begin{gathered}
\psi_{(a)}^{\mu} \psi_{(b) \mu}=\delta_{a b} \psi_{(a)}^{\mu} \psi_{(a) \mu}, \\
\sum_{a=0}^{3} \frac{\psi_{(a)}^{\mu} \psi_{(a)}^{v}}{\psi_{(a)}^{\lambda} \psi_{(a) \lambda}}=\eta^{\mu v}
\end{gathered}
$$

where $\eta^{\mu v}$ is the spacetime Lorentzian metric. Typically, one chooses a scaling for $\psi_{(a)}^{\mu}$ such that

$$
\left(\psi_{(a)}^{\lambda} \psi_{(a) \lambda}\right)^{-1}=\eta^{a a}=\{+1,-1,-1,-1\},
$$

In which case, the properties of Eqs. (A1) and (A2) can be expressed as

$$
\psi_{(a)}^{\mu} \psi_{(b) \mu}=\eta_{a b} \quad \text { and } \quad \sum_{a=0}^{3} \eta^{a a} \psi_{(a)}^{\mu} \psi_{(a)}^{v}=\eta^{\mu \nu}
$$


See section 7 for more details on the scaling symmetry of $\psi_{(a)}^{\mu}$. Note that $a$ is a fictitious Lorentz index, while $\mu$ is a real spacetime tensor index.

\section{Frame independence}

Let $\psi_{(a)}^{\mu}$ be the- basis defined in Sec. 1 and $\phi=\left\{\phi_{1}, \phi_{2}, \ldots\right\}$ be a set of usual local fields with a manifestly covariant Lorentz tensor representation. An action $S[\psi, \phi]$ is frame independent if there exists a change of variables such that

$$
S[\psi, \phi]=\tilde{S}[\Phi]
$$

for some new action $\tilde{S}[\Phi]$ that is independent of $\psi$ (where $\Phi$ is a new set of local fields with a manifestly covariant Lorentz tensor representation). A theory $Z$ with action $S[\psi, \phi]$ is frame independent if there exists some change of variables- such that

$$
Z=\int D \phi e^{i S[\psi, \phi]} \propto \int D \Phi e^{i \tilde{S}[\Phi]}
$$

for some new action $\tilde{S}[\Phi]$ and measure $D \Phi$ that are independent of $\psi$.

\section{Lorentz transformations}

The BTGT index $a$ is a label and not Lorentz tensor index. Therefore the BTGT field $\theta_{a}$ transforms as a scalar and the basis $\psi_{(a)}^{\mu}$ as vectors. Under a Lorentz transformation $x \rightarrow \Lambda x$,

$$
\begin{aligned}
\psi_{(a)}^{\mu} & \rightarrow \Lambda^{\mu}{ }_{v} \psi_{(a)}^{v} \\
\theta_{a}(x) & \rightarrow \theta_{a}\left(\Lambda^{-1} x\right) \\
\phi(x) & \rightarrow \phi\left(\Lambda^{-1} x\right)
\end{aligned}
$$

where $\phi(x)$ is some scalar matter field. The action must be invariant under the Lorentz transformations of Eq. (A7).

\section{Gauge transformations}

Given a matter fields $\phi$ transforming as the fundamental representation of the gauge group $\mathscr{G}$, the BTGT fields $U_{a}$ are defined to transform under a gauge transformation such that $U_{a}^{-1} \phi$ and 
$\phi^{\dagger} U_{a}$ are gauge group singlets. Therefore the gauge transformations of $U_{a}$ are

$$
\begin{gathered}
U_{a}(x) \stackrel{\text { gauge }}{\rightarrow} e^{i \Gamma(x)} U_{a}(x), \\
U_{a}^{-1}(x) \stackrel{\text { gauge }}{\rightarrow} U_{a}^{-1}(x) e^{-i \Gamma(x),}
\end{gathered}
$$

where $U_{a}(x)=e^{i T^{A} \theta_{a}^{A}(x)}$.

\section{BTGT transformations}

Given a set of four scalar BTGT fields $U_{a}$, the BTGT transformation is given by

$$
U_{a}(x) \stackrel{\text { btgt }}{\rightarrow} U_{a}(x) e^{i Z_{a}(x)}
$$

where $Z_{a}(x)=T^{A} Z_{a}^{A}(x)$ satisfies the zero mode constraint

$$
\psi_{(a)}^{\mu} \partial_{\mu} Z_{a}(x)=0 . \quad(\text { no sum over } a)
$$

The BTGT variation is defined to be zero for all other fields:

$$
\delta_{\mathrm{btgt}} \psi_{(a)}^{\mu}=\delta_{\mathrm{btgt}} \phi=0
$$

\section{Permutation symmetry of BTGT labels}

Relabeling symmetry is generated by the exchanges

$$
\left\{H^{a}, U_{a}\right\} \leftrightarrow\left\{H^{b}, U_{b}\right\}
$$

for any $a$ and $b$. In terms of $\psi_{(a)}$ and $U_{a}$ it is

$$
\left\{\eta^{a a}, \psi_{(a)}, U_{a}\right\} \leftrightarrow\left\{\eta^{b b}, \psi_{(b)}, U_{b}\right\}
$$

We impose this condition on BTGT to obtain ordinary gauge theory. One possible complication of this permutation symmetry is that it affects the interpretation of $\psi_{(a)}^{\mu}$ as the Jacobian of a frame change from $x[1]$ to $x[2]$. The swapping of Latin index $a$ on $\eta_{a a}$ must be matched with some swapping of spacetime indices on $\eta_{\mu v}$. When both Latin and Greek indices are free and not summed seems to be the only possible problematic case. However, there was no case found for which this would occur. The issue does not therefore seem important at this time. 


\section{Rescaling symmetry of $\psi_{(a)}^{\mu}$}

Each $\psi_{(a)}^{\mu}$ has an independent rescaling symmetry

$$
\psi_{(a)}^{\mu} \rightarrow \lambda_{a} \psi_{(a)}^{\mu}
$$

for any constant $\lambda_{a} \neq 0$. Note that the properties of $\psi_{(a)}^{\mu}$ given by Eqs. (A1) and (A2) are invariant under the transformation of Eq. (A15). One can normalize the basis fields $\psi_{(a)}^{\mu}$ such that

$$
\psi_{(a)}^{\lambda} \psi_{(a) \lambda}=\eta_{a a}=\{+1,-1,-1,-1\}
$$

by using a rescaling of $\lambda_{a}=1 / \sqrt{\mid \psi_{(a)}^{\lambda} \psi_{(a) \lambda \mid}}$. In that case, there remains a residual parity symmetry of the form

$$
\psi_{(a)}^{\mu} \rightarrow \operatorname{sign}\left(\lambda_{a}\right) \psi_{(a)}^{\mu}
$$

that corresponds to the $P T$ discrete subgroup of the $O(1,3)$ Lorentz group.

\section{Pure gauge basis invariance}

A pure gauge configuration is when $U_{a}=e^{i \Gamma}$, which is gauge equivalent to the identity element $\mathbf{1} \in \mathscr{G}$. When $U_{a}$ is pure gauge, the theory is invariant under a basis change of the form

$$
\psi_{(a)}^{\mu} \rightarrow \sum_{b} \Lambda_{a}^{b} \psi_{(b)}^{\mu}
$$

where $\Lambda_{a}^{b} \in O(1,3)$ is some Lorentz transformation and basis set has been normalized via Eq. (A16).

\section{Appendix B: The only $U_{a}$ dependence of $\mathscr{L}$ is $U_{a} \partial_{(a)} U_{a}^{-1}$ and $\partial_{\mu}\left(U_{a} \partial_{(a)} U_{a}^{-1}\right)$}

In this section we will show that if the action $S=S\left[\psi_{(a)}^{\mu}, U_{a}, \phi\right]$ is invariant under the BTGT symmetry defined in Eq. (A10), then the field dependence of the Lagrangian of that action up to two derivatives of the fields can be expressed as

$$
\mathscr{L}=\mathscr{L}\left(\psi_{(a)}^{\mu}, U_{a} \partial_{(a)} U_{a}^{-1}, \partial_{\mu}\left(U_{a} \partial_{(a)} U_{a}^{-1}\right), \phi, \partial_{\mu} \phi\right)
$$

where $\partial_{(a)} U_{a}^{-1}=\psi_{(a)}^{\mu} \partial_{\mu} U_{a}^{-1}$. 
Let's begin. In terms of all the fields and their derivatives, the most general Lagrangian has field dependence of the form

$$
\mathscr{L}=\mathscr{L}\left(\psi_{(a)}^{\mu}, U_{a}, \partial_{\mu} U_{a}, \partial_{\mu} \partial_{\nu} U_{a}, U_{a}^{-1}, \partial_{\mu} U_{a}^{-1}, \partial_{\mu} \partial_{\nu} U_{a}^{-1}, \phi, \partial_{\mu} \phi\right)
$$

where the space-time dependence is implicit. Since $\partial_{\mu} U_{a}=-U_{a} \partial_{\mu}\left(U_{a}^{-1}\right) U_{a}$, the Lagrangian has field dependence

$$
\mathscr{L}(x)=\mathscr{L}\left(\psi_{(a)}^{\mu}, U_{a}, U_{a}^{-1}, \partial_{\mu} U_{a}^{-1}, \partial_{\mu} \partial_{\nu} U_{a}^{-1}, \phi, \partial_{\mu} \phi\right)
$$

without loss of generality compared to Eq. (B2).

To obtain BTGT singlets in $\mathscr{L}$, one must have pairs of $U_{a}$ and some derivative of $U_{a}^{-1}$, with $U_{a}$ appearing to the left of $U_{a}^{-1}$. These $U_{a}, U_{a}^{-1}$ pairs are the building blocks of making larger BTGT invariants. We can show by exhaustion that each of them can be expressed in terms of $U_{a} \partial_{(a)} U_{a}^{-1}$ and its derivatives. Let us list all such building blocks up to two derivatives acting on $U_{a}$. There are the field independent invariant combinations

$$
\begin{aligned}
\mathbf{1} & =U_{a} U_{a}^{-1}=U_{a}^{-1} U_{a}, \\
\varepsilon_{k_{1} k_{2} \cdots k_{N}} & =\varepsilon_{i_{1} i_{2} \cdots i_{N}}\left(U_{a}\right)_{i_{1} k_{1}}\left(U_{a}\right)_{i_{2} k_{2}} \cdots\left(U_{a}\right)_{i_{N} k_{N}},
\end{aligned}
$$

and the non-trivial invariant

$$
U_{a} \partial_{(a)}\left(U_{a}^{-1}\right)
$$

and its derivative $\partial_{\mu}\left(U_{a} \partial_{(a)} U_{a}^{-1}\right)$. The other invariants are $\partial_{(a)}\left(U_{a}\right) U_{a}^{-1}, U_{a} \partial_{(a)}\left(\partial_{(a)}\left(U_{a}^{-1}\right)\right)$, $\partial_{(a)}\left(U_{a}\right) \partial_{(a)}\left(U_{a}^{-1}\right)$, and $\partial_{(a)}\left(\partial_{(a)}\left(U_{a}\right)\right) U_{a}^{-1}$. Each of these can be expressed in terms of $U_{a} \partial_{(a)} U_{a}^{-1}$ and its derivative:

$$
\begin{gathered}
\partial_{(a)}\left(U_{a}\right) U_{a}^{-1}=-U_{a} \partial_{(a)}\left(U_{a}^{-1}\right) \\
U_{a} \partial_{(a)}\left(\partial_{(a)}\left(U_{a}^{-1}\right)\right)=\partial_{(a)}\left(U_{a} \partial_{(a)}\left(U_{a}^{-1}\right)\right)+U_{a} \partial_{(a)}\left(U_{a}^{-1}\right) U_{a} \partial_{(a)}\left(U_{a}^{-1}\right) \\
\partial_{(a)}\left(U_{a}\right) \partial_{(a)}\left(U_{a}^{-1}\right)=-U_{a} \partial_{(a)}\left(U_{a}^{-1}\right) U_{a} \partial_{(a)}\left(U_{a}^{-1}\right) \\
\partial_{(a)}\left(\partial_{(a)}\left(U_{a}\right)\right) U_{a}^{-1}=-\partial_{(a)}\left(U_{a} \partial_{(a)}\left(U_{a}^{-1}\right)\right)+U_{a} \partial_{(a)}\left(U_{a}^{-1}\right) U_{a} \partial_{(a)}\left(U_{a}^{-1}\right)
\end{gathered}
$$

BTGT invariants are made from these building blocks and can therefore be expressed in terms of $U_{a} \partial_{(a)} U_{a}^{-1}$ and its derivative.

In addition, we have the following combinations that transform as BTGT adjoints $U_{a}^{-1} \partial_{(a)}\left(U_{a}\right)$, $\partial_{(a)}\left(U_{a}^{-1}\right) U_{a}, \partial_{(a)}\left(\partial_{(a)}\left(U_{a}^{-1}\right)\right) U_{a}, \partial_{(a)}\left(U_{a}^{-1}\right) \partial_{(a)}\left(U_{a}\right)$, and $U_{a}^{-1} \partial_{(a)}\left(\partial_{(a)}\left(U_{a}\right)\right)$. These can be 
expressed as

$$
\begin{aligned}
U_{a}^{-1} \partial_{(a)}\left(U_{a}\right) & =U_{a}^{-1}\left(\partial_{(a)}\left(U_{a}\right) U_{a}^{-1}\right) U_{a} \\
\partial_{(a)}\left(U_{a}^{-1}\right) U_{a} & =U_{a}^{-1}\left(U_{a} \partial_{(a)}\left(U_{a}^{-1}\right)\right) U_{a} \\
\partial_{(a)}\left(\partial_{(a)}\left(U_{a}^{-1}\right)\right) U_{a} & =U_{a}^{-1}\left(U_{a} \partial_{(a)}\left(\partial_{(a)}\left(U_{a}^{-1}\right)\right)\right) U_{a} \\
\partial_{(a)}\left(U_{a}^{-1}\right) \partial_{(a)}\left(U_{a}\right) & =-U_{a}^{-1}\left(U_{a} \partial_{(a)}\left(U_{a}^{-1}\right)\right)\left(U_{a} \partial_{(a)} U_{a}^{-1}\right) U_{a} \\
U_{a}^{-1} \partial_{(a)}\left(\partial_{(a)}\left(U_{a}\right)\right) & =U_{a}^{-1}\left(\partial_{(a)}\left(\partial_{(a)}\left(U_{a}\right)\right) U_{a}^{-1}\right) U_{a}
\end{aligned}
$$

All of these BTGT adjoints have the form of

$$
\text { BTGT adjoint }=U_{a}^{-1}(\text { BTGT invariant }) U_{a}
$$

Since $\mathscr{L}$ must be a BTGT invariant, the BTGT adjoint objects must eventually appear in a group trace composed only of BTGT adjoint objects [22]. Since $U_{a}$ is the only field with a BTGT charge, these are the only BTGT adjoint objects that appear in $\mathscr{L}$. Therefore, they can only appear with each other in a trace. Inside the trace, they become equivalent to BTGT invariants:

$$
\begin{aligned}
\operatorname{Tr}[(\text { BTGT adj. }) \cdots(\text { BTGT adj. })] & =\operatorname{Tr}\left[U_{a}^{-1}(\text { BTGT inv. }) U_{a} \cdots U_{a}^{-1}(\text { BTGT inv. }) U_{a}\right] \\
& =\operatorname{Tr}[(\text { BTGT inv. }) \cdots(\text { BTGT inv. })]
\end{aligned}
$$

As a result, the only non-trivial $U_{a}$ dependence of $\mathscr{L}$ is on $U_{a} \partial_{(a)}\left(U_{a}^{-1}\right)$ and its derivative $\partial_{\mu} U_{a} \partial_{(a)}\left(U_{a}^{-1}\right)$.

\section{Appendix C: Frame independence for non-renormalizable terms}

When vierbein field $G^{\mu}{ }_{v}$ is parameterized as Eq. (23) in terms of $H^{a}$ and $U_{a}$, there are certain transformations of $H^{a}$ and $U_{a}$ that keep $G^{\mu}{ }_{v}$ invariant. These symmetries include the relabeling permutation symmetry of Eq. (A13), and the $\psi_{(a)}^{\mu}$ scaling/parity symmetry of Eqs. (A15) and (A17), and the pure gauge basis invariance defined in Appendix A. Another symmetry that would keep Eq. (23) invariant is

$$
H^{a} \rightarrow e^{\phi_{a}} H^{a}, \quad U_{a} \rightarrow e^{-\phi_{a}} U_{a}, \quad U_{a}^{\dagger} \rightarrow e^{-\phi_{a}} U_{a}^{\dagger}
$$

where $\phi_{a}$ is a real global parameter. This scaling symmetry is designed so that $H^{a}$ appears only in the form of $H^{a} U_{a}$ or $H^{a} U_{a}^{\dagger}$ (no sum over $a$ ). 
The symmetry of Eq. (C1) prevents frame dependent terms like Eqs. (96) and (97) from emerging in any effective action potential. However, the scaling also corresponds to a deformation of the properties of both $H^{a}$ and $U_{a}$ as previously defined. For example, the inverse to $U_{a}$ would satisfy

$$
U_{a}^{-1}=\operatorname{Tr}\left(H^{a}\right)^{2} U_{a}^{\dagger}
$$

instead of $U_{a}^{-1}=U_{a}^{\dagger}$. In addition, $\theta_{a}=\theta_{a}^{A} T^{A}$ is deformed from the Lie algebra under the scaling of Eq. (C1) by

$$
\theta_{a}^{A} T^{A} \rightarrow \theta_{a}^{A} T^{A}+i \phi_{a} \mathbf{1}
$$

where $\mathbf{1}$ is multiplicative algebra identity such that $\left[T^{A}, \mathbf{1}\right]=0$. The imaginary component of Eq. ([C3) is an analytic continuation of the field $\theta_{a}$. Covariant derivatives would be modified to

$$
D_{\mu}(\cdot)=\sum_{a} \operatorname{Tr}\left(H^{a}\right) U_{a}\left(H^{a}\right)_{\mu}^{\lambda} \partial_{\lambda}\left(U_{a}^{\dagger} \cdot\right)
$$

which is obtained by replacing $U_{a}^{-1}$ with Eq. (C2) and $H^{a}$ with $H^{a} / \operatorname{Tr}\left(H^{a}\right)$. The same replacements should be consistently done in the BTGT and gauge invariant Lagrangian.

[1] N. Arkani-Hamed and J. Trnka, The Amplituhedron, JHEP 10 (2014) 030, [1312.2007].

[2] N. Arkani-Hamed, T.-C. Huang and Y.-t. Huang, Scattering Amplitudes For All Masses and Spins, 1709.04891

[3] S. D. Badger, E. W. N. Glover, V. V. Khoze and P. Svrcek, Recursion relations for gauge theory amplitudes with massive particles, JHEP 07 (2005) 025, [hep-th/0504159].

[4] H. Elvang and Y.-t. Huang, Scattering Amplitudes, 1308.1697.

[5] J. M. Henn and J. C. Plefka, Scattering Amplitudes in Gauge Theories, Lect. Notes Phys. 883 (2014) pp.1-195.

[6] N. Christensen and B. Field, Constructive standard model, Phys. Rev. D98 (2018) 016014. [1802.00448].

[7] E. Witten, Anti-de Sitter space and holography, Adv. Theor. Math. Phys. 2 (1998) 253-291. [hep-th/9802150].

[8] O. Aharony, S. S. Gubser, J. M. Maldacena, H. Ooguri and Y. Oz, Large N field theories, string theory and gravity, Phys. Rept. 323 (2000) 183-386, [hep-th/9905111].

[9] C.-N. Yang and R. L. Mills, Conservation of Isotopic Spin and Isotopic Gauge Invariance, Phys. Rev. 96 (1954) 191-195. 
[10] E. S. Abers and B. W. Lee, Gauge Theories, Phys. Rept. 9 (1973) 1-141.

[11] C. Itzykson and J. B. Zuber, Quantum Field Theory. International Series In Pure and Applied Physics. McGraw-Hill, New York, 1980.

[12] A. M. Polyakov, Gauge Fields and Strings, Contemp. Concepts Phys. 3 (1987) 1-301.

[13] G. F. Sterman, An Introduction to quantum field theory. Cambridge University Press, 1993.

[14] G. 't Hooft, Under the spell of the gauge principle, Adv. Ser. Math. Phys. 19 (1994) 1-683.

[15] S. Weinberg, The quantum theory of fields. Vol. 2: Modern applications. Cambridge University Press, 2013.

[16] D. J. H. Chung and R. Lu, Basis tensor gauge theory: Reformulating gauge theories with basis tensor fields, Phys. Rev. D94 (2016) 105016, [1609.03679].

[17] D. J. H. Chung, Ward Identity and Basis Tensor Gauge Theory at One Loop, Phys. Rev. D97 (2018) 125003, [1712.10118].

[18] E. Basso and D. J. H. Chung, Non-Abelian basis tensor gauge theory, 1905.08363.

[19] A. Kovner and B. Rosenstein, New look at QED in four-dimensions: The Photon as a Goldstone boson and the topological interpretation of electric charge, Phys. Rev. D49 (1994) 5571-5581, [hep-th/9210154].

[20] D. Gaiotto, A. Kapustin, N. Seiberg and B. Willett, Generalized Global Symmetries, JHEP 02 (2015) 172, [1412.5148].

[21] D. M. Hofman and N. Iqbal, Goldstone modes and photonization for higher form symmetries, SciPost Phys. 6 (2019) 006, [1802.09512].

[22] A. J. Mountain, Invariant tensors and casimir operators for simple compact lie groups, Journal of Mathematical Physics 39 (1998) 5601-5607, [https://doi .org/10.1063/1.532552]. 